\title{
Transketolase promotes colorectal cancer metastasis through regulating AKT phosphorylation
}

\author{
Minle $\mathrm{Li}^{1,4}$, Xue Zhao ${ }^{1,4}$, Hongmei Yong ${ }^{2,4}$, Jian $\mathrm{Xu}^{1}$, Pengfei $\mathrm{Qu}^{1}$, Shuxi Qiao ${ }^{1}$, Pingfu Hou ${ }^{1}$, Zhongwei $\mathrm{Li}^{1}$, Sufang $\mathrm{Chu}^{1}$, \\ Junnian Zheng iD $^{1,3 凶}$ and Jin Bai iD $^{1,3}$
}

(c) The Author(s) 2022

Transketolase (TKT) which is an important metabolic enzyme in the pentose phosphate pathway (PPP) participates in maintaining ribose 5-phosphate levels. TKT is necessary for maintaining cell growth. However, we found that in addition to this, TKT can also affect tumor progression through other ways. Our previous study indicate that TKT could promote the development of liver cancer by affecting bile acid metabolism. And in this study, we discovered that TKT expression was remarkably upregulated in colorectal cancer, abnormal high expression of TKT is associated with poor prognosis of colorectal cancer. Additionally, TKT promoted colorectal cancer cell growth and metastasis. Further study demonstrated that TKT interacted with GRP78 and promoted colorectal cancer cell glycolysis through increasing AKT phosphorylation, thereby enhancing colorectal cancer cell metastasis. Thus, TKT is expected to become an indicator for judging the prognosis of colorectal cancer, and provide a theoretical basis for drug development of new treatment targets for colorectal cancer.

Cell Death and Disease (2022)13:99; https://doi.org/10.1038/s41419-022-04575-5

\section{INTRODUCTION}

One of the characteristics of malignant tumors is enhanced aerobic glycolysis [1]. Emerging studies have revealed that glycolysis is positively correlated with the malignant progression of cancer $[2,3]$. It is evident that lactate promotes tumor growth and metastasis [4-6]. Thus, targeting glycolysis remains attractive for cancer therapy.

Colorectal cancer (CRC) is a malignant tumor of the digestive system [7, 8], and about half of patients with CRC die due to tumor metastasis [9]. Therefore, finding a potential therapeutic target for CRC metastasis is important for clinical treatment.

The transketolase (TKT) family includes three members of TKT, TKTL1, and TKTL2. In recent years, a lot of research is focused on TKTL1, and find that TKTL1 is highly expressed in lung cancer, cervical cancer, esophageal squamous cell carcinoma. [10-13]. TKTL1 is positively associated with tumor development and poor prognosis [14-16]. However, few studies have focused on TKT, actually, in most tumors, TKT is the highest-expressing member of the transketolase family [17]. In hepatocellular carcinoma (HCC), TKT knockdown causes R5P accumulation but inhibits cell proliferation, the explanation for this phenomenon is that the absence of TKT results in a decrease in NADPH, which disrupts the cell's redox balance and causes an increase in ROS [18]. Some studies have pointed out that high expression of TKT in cervical cancer and pancreatic cancer enhance the PPP activity, thereby providing raw materials for the rapid growth and proliferation of tumor cells $[19,20]$. In the above studies, TKT played its role through the PPP. However, in recent years, TKT's functions other than promoting R5P generation have attracted people's attention. For example, in breast cancer, TKT promotes metastasis through regulatinga-Ketoglutarate signaling pathway [21], and it has been shown that TKT enters the nucleus and activates the EGFR pathway, thereby promoting proliferation, viability, and migration of liver cancer [22]. Furthermore, our previous research also confirms that TKT transports into the nuclear to inhibit FXR promoter activity, affects liver bile acid metabolism, and promotes liver cancer [23]. Nevertheless, the function of TKT and related mechanisms in CRC are currently unclear.

In the present study, we used tissue microarrays (TMAs) of CRC patients with prognostic information to explore the function of TKT in CRC. We evaluated that TKT could be used as an independent prognostic indicator for CRC treatment, and we confirmed that TKT promoted CRC cells growth and metastasis. The function and related molecular mechanism of TKT in CRC metastasis were firstly elucidated in this study.

\section{RESULTS}

TKT was highly expressed in CRC and cell lines and indicated poor prognosis

We tested TKT expression in 10 pairs of colorectal cancer and adjacent samples. The data demonstrated that compared with adjacent normal tissues, TKT expression was significantly upregulated in tumor tissues, and increased TKT expression was also detected in the Gene Expression Profiling Interactive Analysis (GEPIA) (Fig. 1A, B). Consistently, compared with FHC (normal

\footnotetext{
${ }^{1}$ Cancer Institute, Xuzhou Medical University, Xuzhou, Jiangsu, China. ${ }^{2}$ Department of Oncology, The Affiliated Huai'an Hospital of Xuzhou Medical University and The second People's Hospital of Huai'an, Huai'an 223002, China. ${ }^{3}$ Center of Clinical Oncology, Affiliated Hospital of Xuzhou Medical University, Xuzhou, Jiangsu, China. ${ }^{4}$ These authors contributed equally: Minle Li, Xue Zhao, Hongmei Yong. ${ }^{凶}$ email: jnzheng@xzhmu.edu.cn; bj@xzhmu.edu.cn

Edited by Professor Junnian Zheng
}

Received: 14 July 2021 Revised: 9 January 2022 Accepted: 19 January 2022

Published online: 02 February 2022 
A

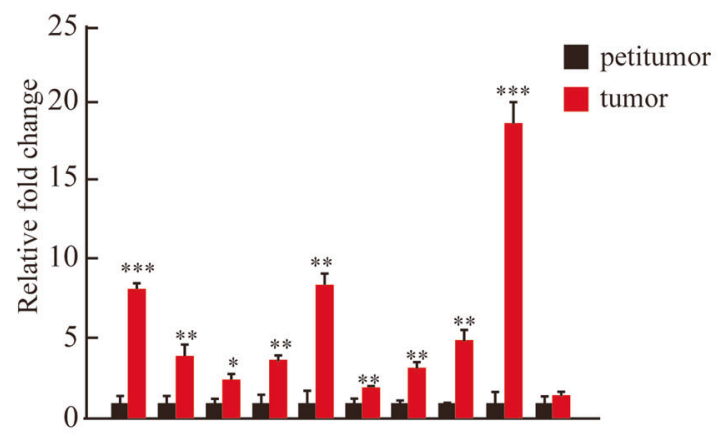

C paracancerous tissue

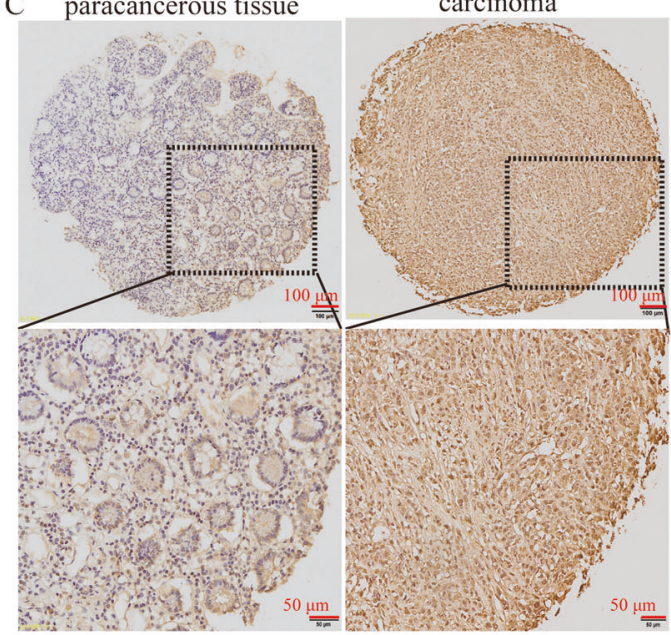

E

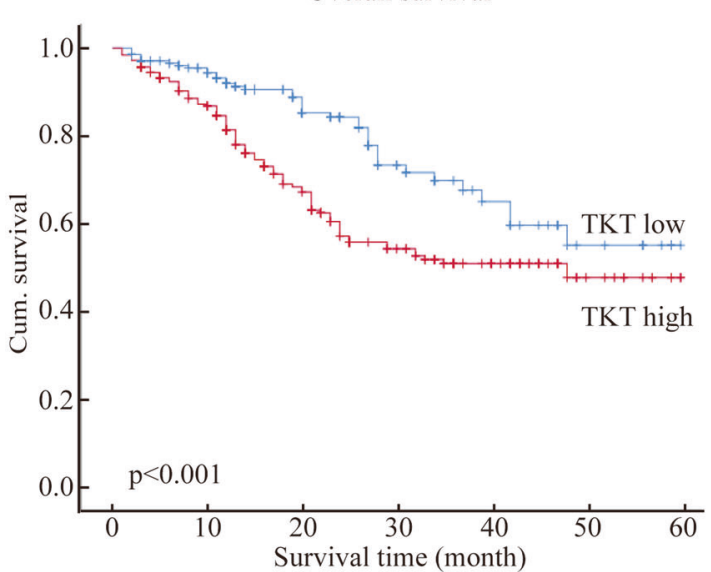

$\mathrm{D}$

$\mathrm{F}$
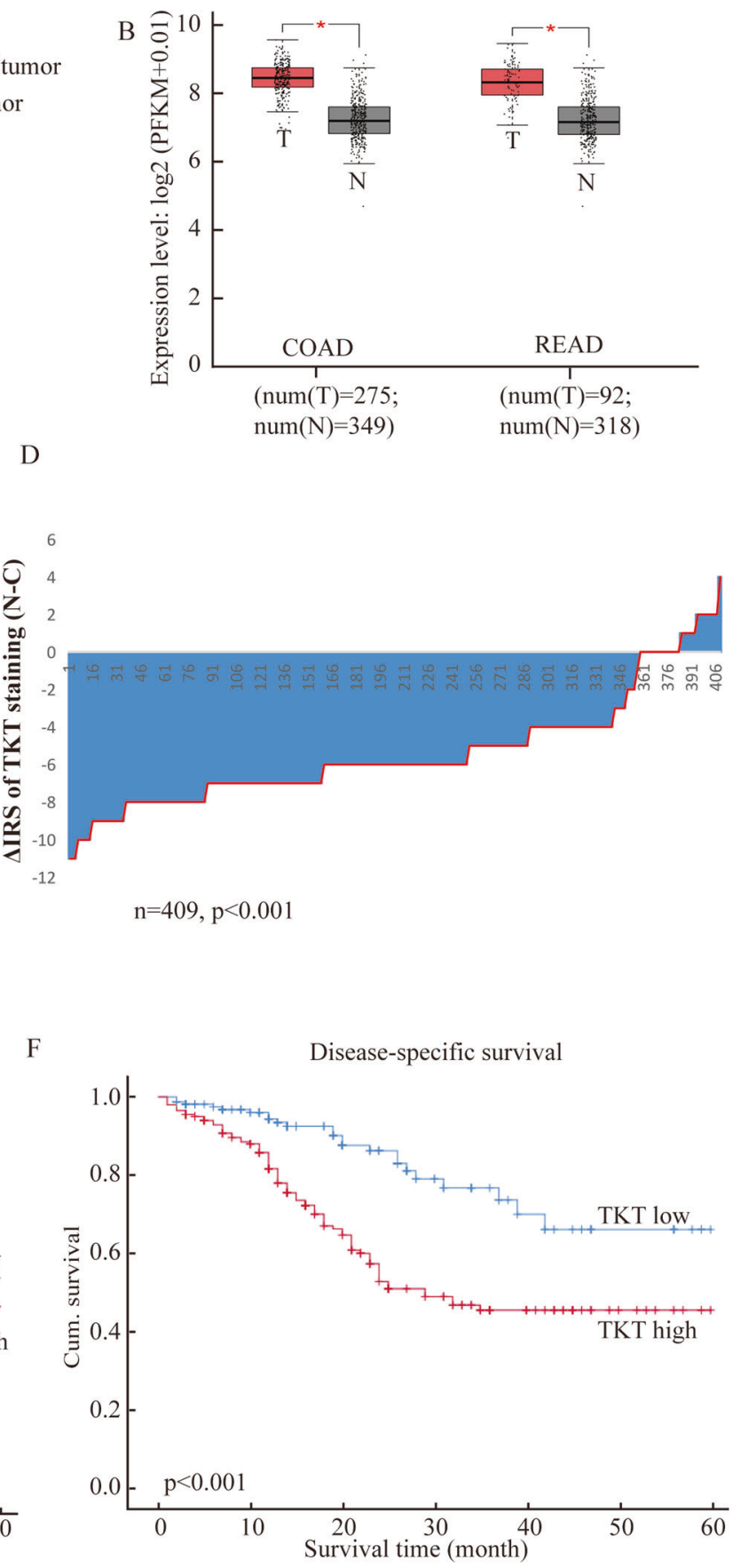

Fig. 1 TKT is elevated in CRC, and is related to poor OS and DFS in CRC. (A) Detection of TKT expression in 10 pairs of colorectal cancer CRC and adjacent tissues. (B) In the Gene Expression Profiling Interactive Analysis (GEPIA), TKT expression was high in CRC. (C) TKT immunostaining in TMAs. Note: magnification $\times 100$. (D) Distribution of TKT staining intensity difference in tumor and tumor-adjacent tissues. Note: N, tumoradjacent tissues; $C$, tumor tissues, $P<0.001$. (E) High TKT expression is regulated to poorer overall survival of patients with $C R C(P<0.001)$. $(F)$ High TKT expression is regulated to poorer disease-specific survival of colorectal carcinoma patients $(P<0.001$, log-rank test).

colorectal epithelial cell), TKT expression was higher in CRC cell lines (HT-29, DLD1, LOVO, SW620, HCT116, SW480) (Fig. S1A).

To clarify the relationship between TKT and the prognosis of CRC, we used immunohistochemical $(\mathrm{IHC})$ analysis to evaluate the level of TKT expression in 464 (81.7\%) of 568 CRC samples and 401 (70.6\%) of 568 nontumor tissues. The expression of TKT in tumor tissues of patients with CRC is significantly higher than adjacent tissues (Fig. 1C). Next, we analyzed the paired cancer and para-cancerous tissues of 409 patients, and the data suggested that TKT expression was significantly increased in cancerous tissue (Fig. 1D).
To further clarify the significance of TKT in the clinical treatment of $\mathrm{CRC}$, we used Fisher's exact test to detect the correlation between TKT expression and clinicopathological characteristics of CRC. Immunoreactivity score (IRS) $1-6$ were classified as TKT low expression, and IRS 8-12 were classified as TKT high expression. The data showed that TKT was low in 45\% (209/464) and high in $55 \%$ (255/464) of CRC tissues. The obvious positive correlation with the high expression of TKT were tumor lymph node metastasis $(P<0.001)$ and tumor node metastasis (TNM) stage $(P<0.001)$. However, TKT expression had no correlation with age, 
gender, depth of invasion, tumor diameter, or differentiation (Table 1).

Kaplan-Meier survival analysis demonstrated that patients with higher TKT expression had poorer overall survival (Fig. 1E). Moreover, we used the KM-plotter to do bioinformatics analysis, the result also showed that CRC patients with higher TKT expression had shorter disease-specific survival (Fig. 1F).

To confirm whether TKT can be an independent indicator for judging the prognosis of CRC, Univariate Cox regression was used to analyze the value of TKT in the prognostic evaluation of CRC. The result showed that TKT expression, lymph node metastasis (LNM), and TNM stage, were the significant prognostic indicators for the OS of CRC patients. Moreover, we used univariate Cox

Table 1. Relationship between TKT expression and clinicopathological features of CRC patients.

\begin{tabular}{|c|c|c|c|}
\hline \multirow[t]{2}{*}{ Variables } & \multicolumn{3}{|c|}{ TKT expression ( $n=464$ cases) } \\
\hline & Low (\%) & High (\%) & $P^{a}$ \\
\hline All patients & $209(45)$ & $255(55)$ & \\
\hline Age (years) & & & 0.454 \\
\hline$<63$ & $105(43)$ & $137(57)$ & \\
\hline$\geq 63$ & $104(47)$ & $118(53)$ & \\
\hline Gender & & & 0.878 \\
\hline Males & $119(45)$ & $147(55)$ & \\
\hline Females & $90(45)$ & $108(55)$ & \\
\hline Depth of invasion & & & 0.695 \\
\hline $\mathrm{T} 1 / \mathrm{T} 2$ & $134(46)$ & $159(54)$ & \\
\hline $\mathrm{T} 3 / \mathrm{T} 4$ & $75(44)$ & $96(56)$ & \\
\hline Lymph node metastasis & & & $<0.001$ \\
\hline NO & $158(54)$ & $137(46)$ & \\
\hline N1/N2/N3 & $51(30)$ & $118(70)$ & \\
\hline Distant metastasis & & & 0.360 \\
\hline Mo & $198(45)$ & $246(55)$ & \\
\hline M1 & $11(55)$ & $9(45)$ & \\
\hline TNM stage & & & $<0.001$ \\
\hline I/II & $158(56)$ & $125(44)$ & \\
\hline III/ IV & $51(28)$ & $130(72)$ & \\
\hline Tumor diameter & & 0.982 & \\
\hline$<5 \mathrm{~cm}$ & $151(45)$ & $184(55)$ & \\
\hline$\geq 5 \mathrm{~cm}$ & $58(45)$ & $71(55)$ & \\
\hline Differentiation & & & 0.658 \\
\hline Poor & $30(48)$ & $33(52)$ & \\
\hline Moderate/high & $179(45)$ & $222(55)$ & \\
\hline
\end{tabular}

regression analysis to find that in the disease-specific survival (DFS) of CRC patients, TKT expression, LNM, and TNM stage were the significant prognostic indicators in the disease-specific survival (DFS) of CRC patients (Table 2). Our data confirmed that TKT high expression may serve as a potential independent prognostic indicator in CRC.

\section{TKT promoted CRC cells proliferation}

Because previous results indicated that TKT affected the malignancy of colorectal cancer, next, we wanted to explore the function of TKT in CRC. We established SW480 and HCT116 stable cell lines with TKT overexpression or knockdown. Then, we used the cell counting kit- 8 assays (CCK-8) to examine the effect of TKT on proliferation. The result suggested that overexpression of TKT significantly promoted cell proliferation. Conversely, knockdown of TKT alleviated cell proliferation (Fig. 2A-H). Moreover, we used RTCA and colony formation assays to further confirm that TKT promoted colorectal cancer cells proliferation (Fig. 2I-L). Furthermore, the xenograft experiments showed that the tumor volume of TKT overexpression group was notably bigger than the control group (Fig. $2 \mathrm{M}, \mathrm{N}$ ). In summary, these results demonstrated that TKT stimulated the growth of CRC cells.

\section{TKT promoted metastasis of CRC cells}

Then, we investigated whether TKT affects the migration and invasion ability of CRC cells. The transwell assays dispalyed that TKT overexpression significantly enhanced migration and invasion ability (Fig. 3A, C), the wound-healing assay also confirmed this phenomenon (Fig. 3E, G). In contrast, the migration and invasion ability of cells weakened with the decrease of TKT expression (Fig. $3 B, D, F, H)$. Furthermore, we observed the same phenomenon in HCT116 via RTCA assay (Fig. S2A-D).

We used tail vein injection of lung metastasis model to test the role of TKT in colorectal cell metastasis in vivo. $1 \times 10^{6}$ HCT116 stable cells with TKT overexpression or knockdown were injected to 8-week-old nude mice, and the lung metastases was detected after 2 months. Compared with the control group, the fluorescence intensity of the TKT overexpression group was stronger and more metastatic foci formed in the lungs (Fig. 4A, C), but TKT knockdown significantly reduced the fluorescence intensity and the number of metastatic foci (Fig. 4B, D).

Angiogenesis plays an important role in tumor growth and metastasis [24]. Because our data suggested that high TKT expression was an essential factor for cancer metastasis, we studied the role of TKT in CRC angiogenesis. We used HUVECs to do blood vessel formation experiments. And we found that the number of complete tubular structures was increased in the medium collected from TKT overexpression cells and reduced in the medium collected from TKT knockdown cells compared with the corresponding controls (Fig. S3A-D).

Epithelial mesenchymal-transition (EMT) can reduce cell-cell adhesion, promote cell migration and invasion, thereby promoting

Table 2. Univariate Cox regression analysis of TKT expression and clinicopathologic variables predicting the survival of CRC patients.

\begin{tabular}{|c|c|c|c|c|c|c|}
\hline \multirow[t]{2}{*}{ Variable* } & \multicolumn{3}{|c|}{ Overall survival } & \multicolumn{3}{|c|}{ Disease-specific survival } \\
\hline & Hazard ratio & $95 \% \mathrm{Cl}^{\dagger}$ & $p^{*}$ & Hazard ratio & $95 \% \mathrm{Cl}^{\dagger}$ & $P^{*}$ \\
\hline TKT & 1.981 & $1.365-2.875$ & $<0.001$ & 1.881 & $1.277-2.771$ & 0.001 \\
\hline Gender & 1.441 & $1.028-2.021$ & 0.034 & 1.476 & $1.003-2.173$ & 0.048 \\
\hline LNM & 1.756 & $1.253-2.462$ & 0.001 & 1.970 & $1.336-2.904$ & 0.001 \\
\hline TNM stage & 1.880 & $1.341-2.635$ & $<0.001$ & 1.881 & $1.277-2.771$ & 0.001 \\
\hline Tumor diameter & 1.114 & $0.768-1.617$ & 0.570 & 0.985 & $0.635-1.528$ & 0.945 \\
\hline Depth of invasion & 1.523 & $1.081-2.147$ & 0.016 & 1.449 & $0.979-2.146$ & 0.064 \\
\hline
\end{tabular}


A

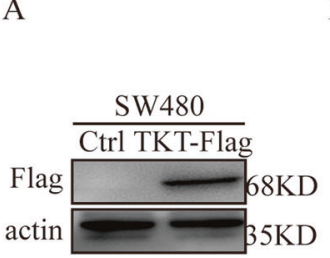

B

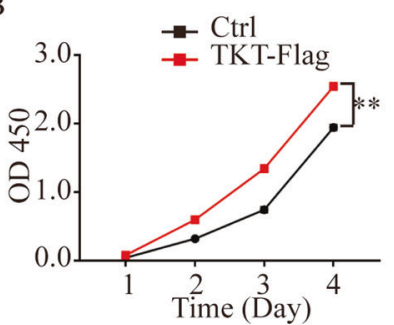

C
D

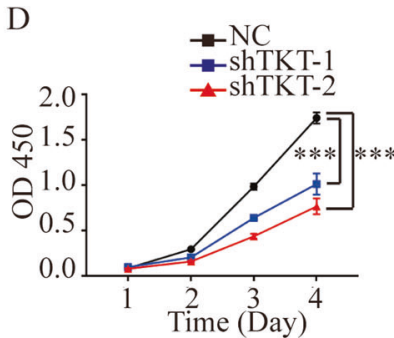

E

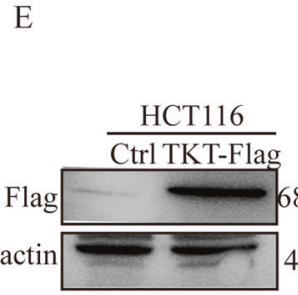

F

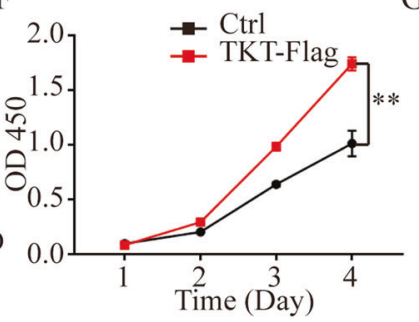

G
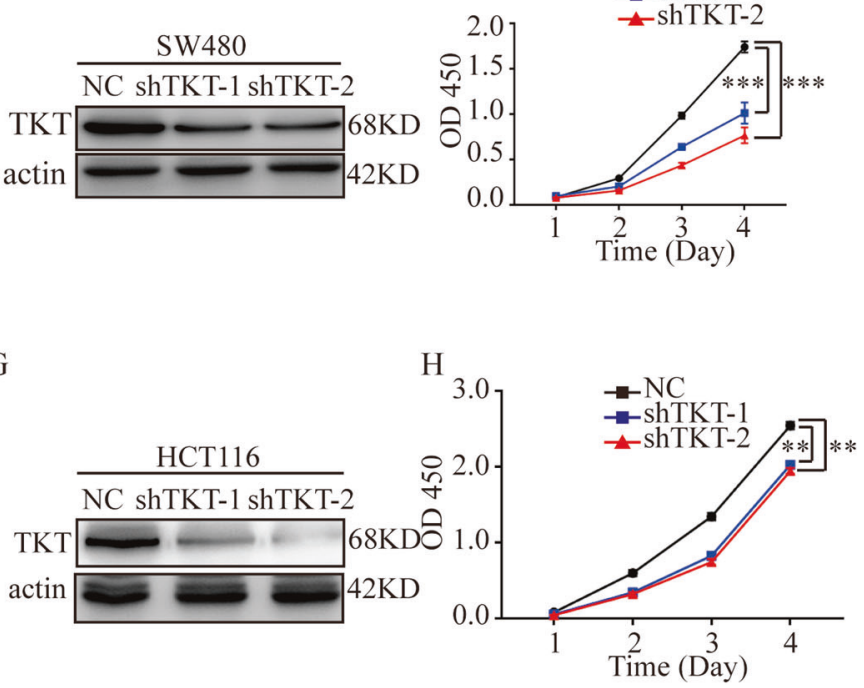

I

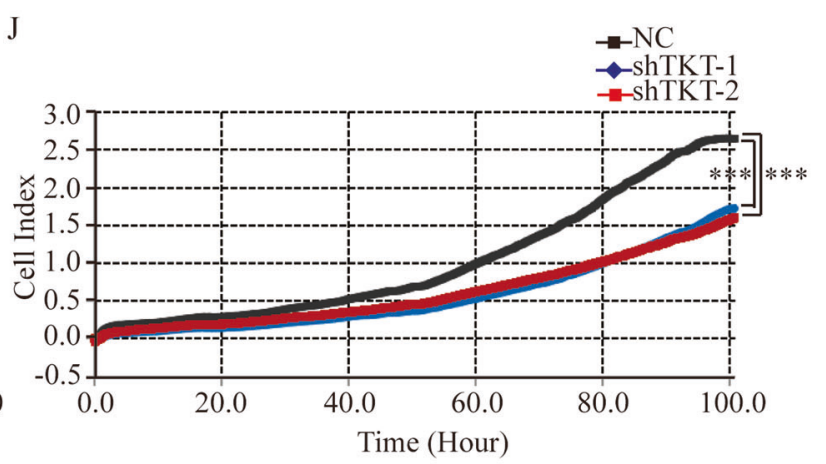

K
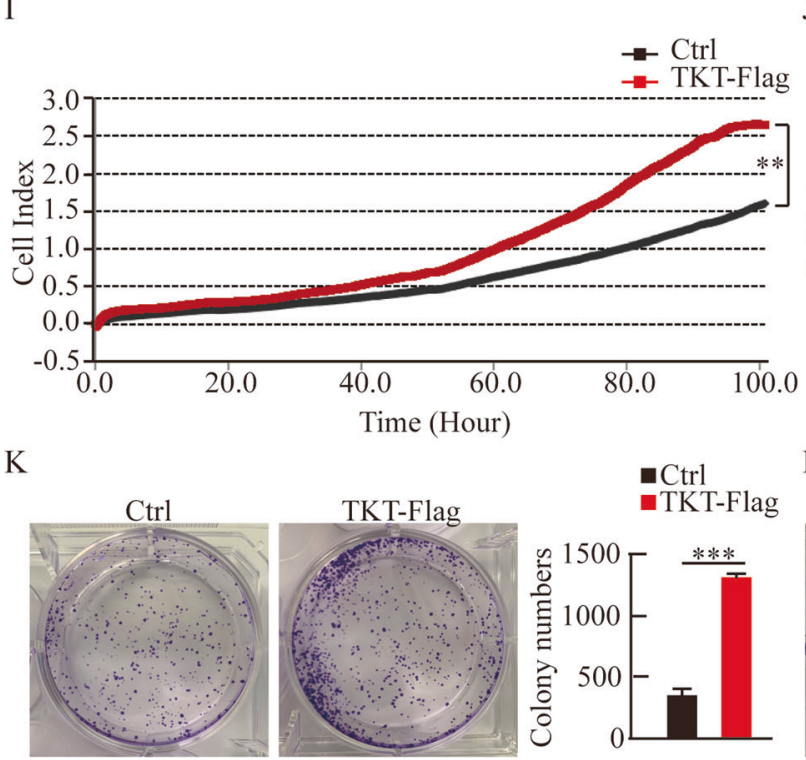

Ctrl
-TKT-Flag

L
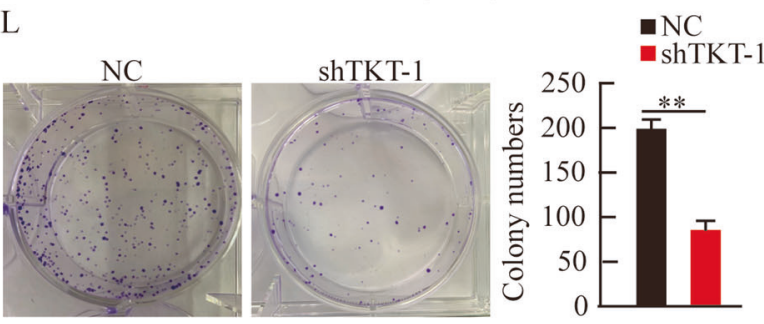

M

$\mathrm{N}$
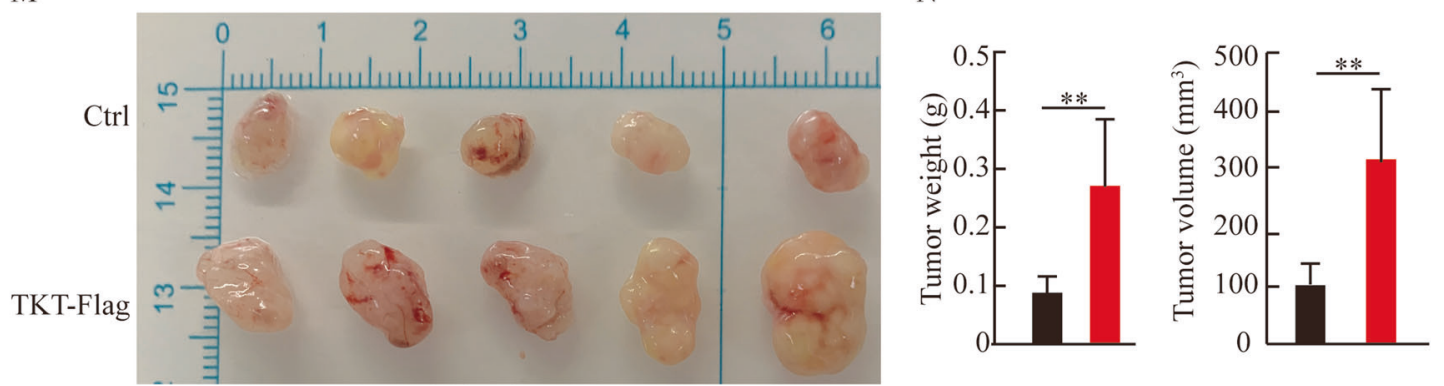

- $\mathrm{NC}$

- shTKT-1

Fig. 2 TKT promotes CRC cell proliferation. (A) Overexpression of TKT was verified in SW480. (B) TKT overexpression enhanced SW480 proliferation ability. (C) Knockdown of TKT was confirmed in SW480. (D) TKT knockdown significantly reduced SW480 proliferation. (E) Overexpression of TKT was confirmed in HCT116. (F) TKT overexpression enhanced HCT116 proliferation ability. (G) Knockdown of TKT was confirmed in HCT116. (H) TKT knockdown significantly reduced HCT116 proliferation. (I) RTCA indicated that TKT overexpression enhanced HCT116 proliferation ability. (J) RTCA indicated that TKT knockdown inhibited HCT116 proliferation. (K-L) Colony formation assays. ${ }^{*} P<0.05$, ${ }^{* *} P<0.01,{ }^{* *} P<0.001$. (M-N) Evaluated the role of TKT in tumor formation by measuring tumor volume and weight $\left({ }^{* *} p<0.01\right)$.

tumor metastasis [25]. We found that TKT overexpression promoted EMT markers (N-cadherin, Vimentin, and Snail) expression but suppressed the level of E-cadherin. By contrast, TKT knockdown upregulated E-cadherin expression and inhibited N-cadherin, Vimentin, and Snail expression (Fig. 4E, F). These data strongly proved that TKT promoted CRC migration, invasion, and metastasis. 

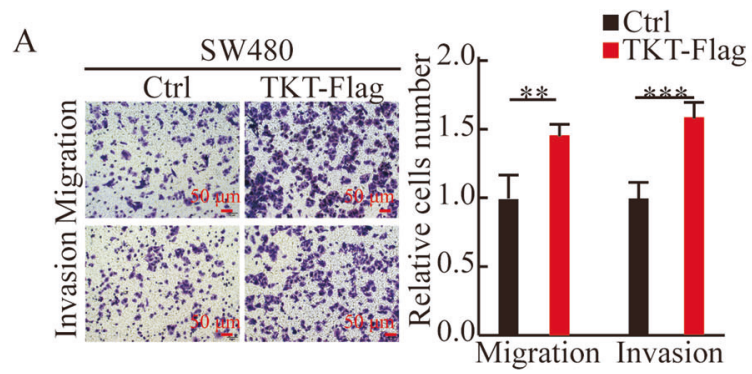

C

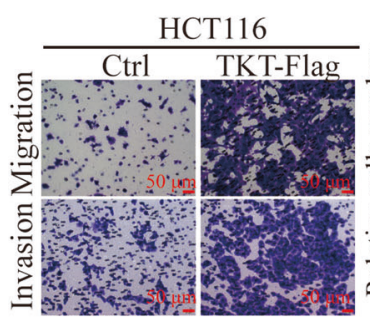

$\mathrm{E}$
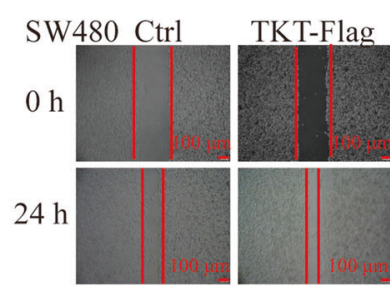

G
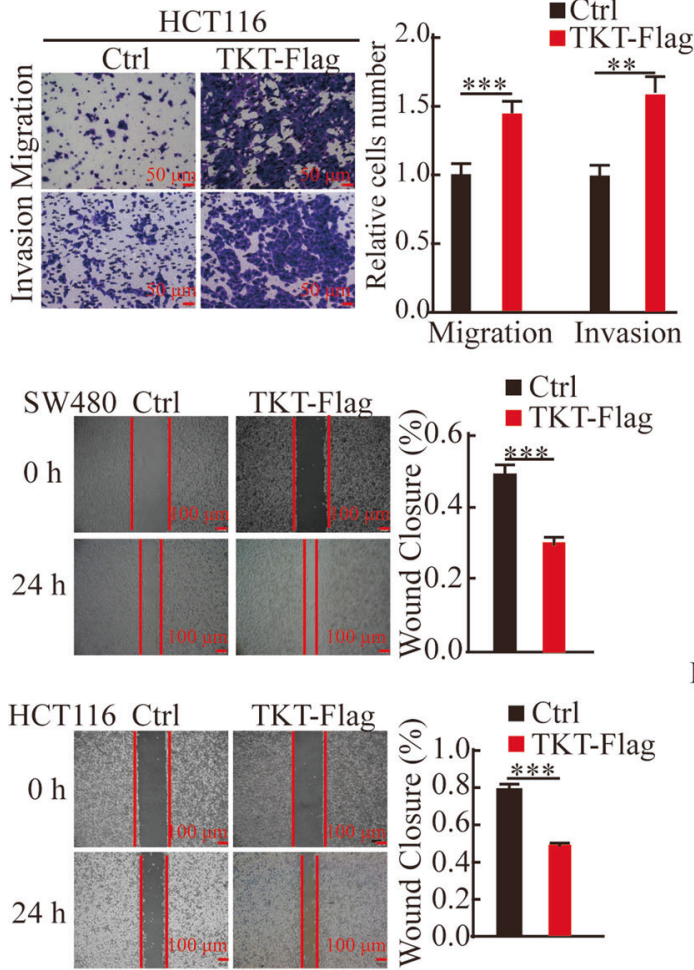

$\square \mathrm{Ctrl}$

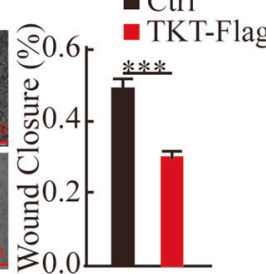

$\mathrm{H}$

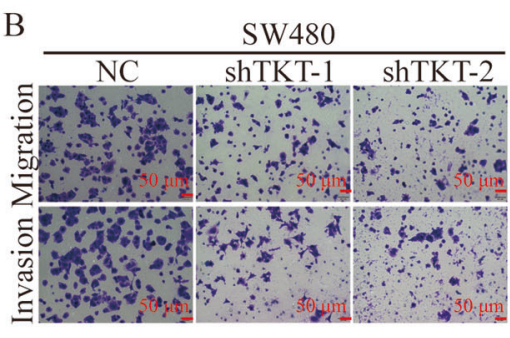

$\mathrm{D}$

SW480 NC
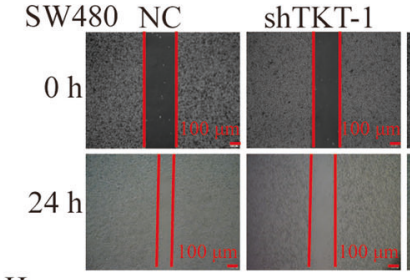

HCT116 NC

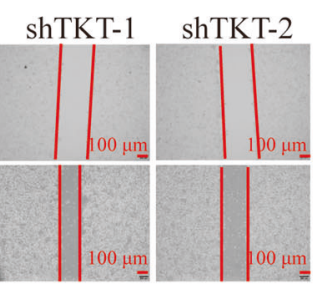

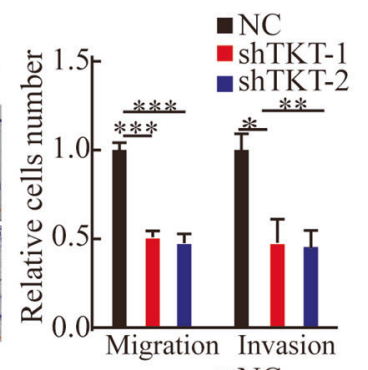
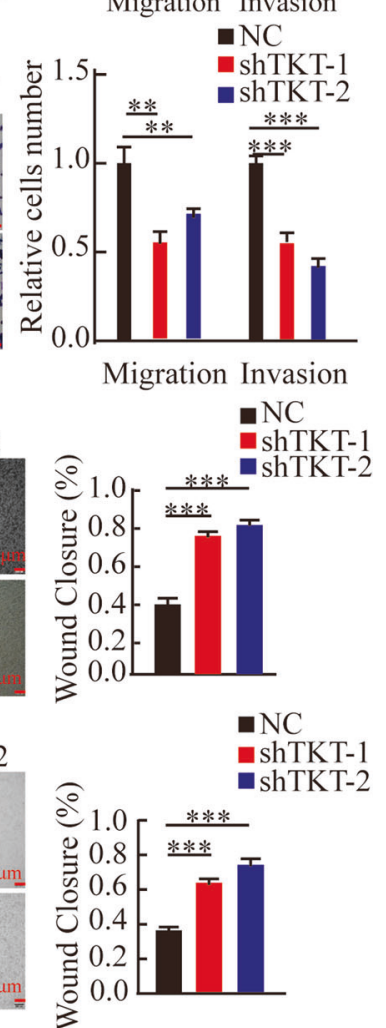

Fig. 3 TKT promoted metastasis of CRC cells. (A-D) Migration and invasion detection of SW480 and HCT116 cells with TKT overexpression and knockdown. (E-H) Wound-healing assays. ${ }^{*} P<0.05,{ }^{* *} P<0.01,{ }^{* * *} P<0.001$.

\section{TKT promoted CRC cells metastasis through activating aerobic glycolysis}

Tumor metastasis is usually accompanied by high-intensity glycolysis. Our results showed that TKT significantly increased aerobic glycolysis, glucose consumption, lactic acid production of CRC cells as expected (Fig. 5A-C). Conversely, TKT knockdown reduced aerobic glycolysis, glucose consumption, lactic acid (Fig. 5D-F). In addition, we found that TKT overexpression also resulted in increased fructose 6-phosphate (F6P) production, which is the intermediate of the non-oxidative phase (Fig. 5G, H). It is well known that F6P can enter the glycolytic pathway and then be metabolized, this result further confirmed that TKT overexpression could promote glycolysis. Moreover, we detected the expression of genes regulating glucose metabolism. The data showed that TKT overexpression enhanced Glut1 and LDHA expression while knockdown of TKT inhibited these genes (Fig. 5I).

In order to test whether TKT's promotion of colorectal cancer metastasis is dependent on glycolysis, we used galactose which induced cells to survive on ATP provided by mitochondrial respiration instead of glucose. We found that decreased glycolysis caused by galactose significantly attenuated migration and invasion promoted by TKT overexpression (Fig. 5J-M). And the increased expression of EMT marker induced by TKT overexpression was also reversed (Fig. S4A). These results implied that TKT may exert its function of promoting metastasis in CRC cells through activating aerobic glycolysis.

\section{TKT promoted aerobic glycolysis of CRC cells by promoting AKT phosphorylation}

To investigate the possible mechanism of TKT in CRC metastasis, we determined the possible genes from the previous mass spectrometry results [23] and focused on GRP78 because GRP78 promotes tumor growth and migration via regulating AKT phosphorylation [26]. We suspected that TKT regulated AKT phosphorylation through interacting with GRP78 and then promoted aerobic glycolysis, thereby facilitated CRC cell metastasis.

Next, we evaluated the effect of TKT on AKT phosphorylation. The results showed that in SW480 and HCT116, the overexpression of TKT promoted AKT phosphorylation, and TKT reduction decreased AKT phosphorylation (Fig. 6A). To determine whether AKT phosphorylation has an effect on cell metabolism, we used drugs (LY294002) to inhibit AKT phosphorylation (Fig. 6B). We found that when AKT phosphorylation was inhibited, the enhanced aerobic glycolysis induced by elevated TKT expression was reversed (Fig. 6C-G). In addition, we showed that inhibition of AKT phosphorylation significantly reduced cell migration and invasion ability induced by TKT high expression (Fig. $6 \mathrm{H}$, I). These 


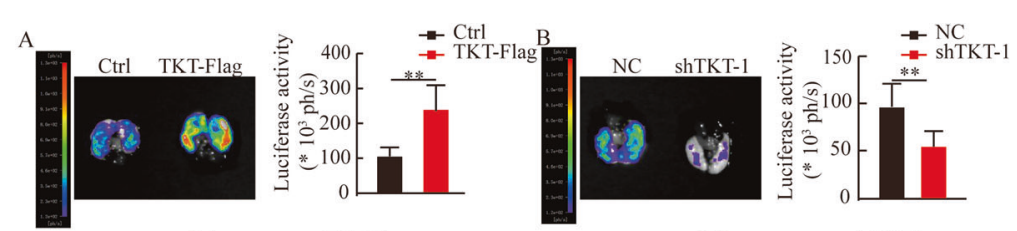

$\mathrm{C}$

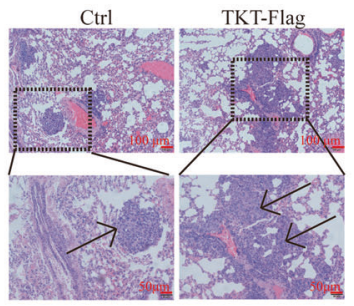

D

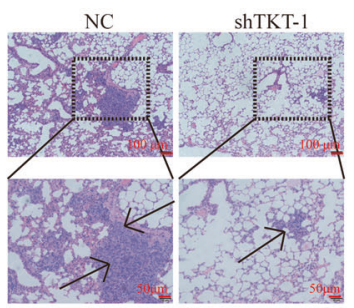

E

$\mathrm{F}$
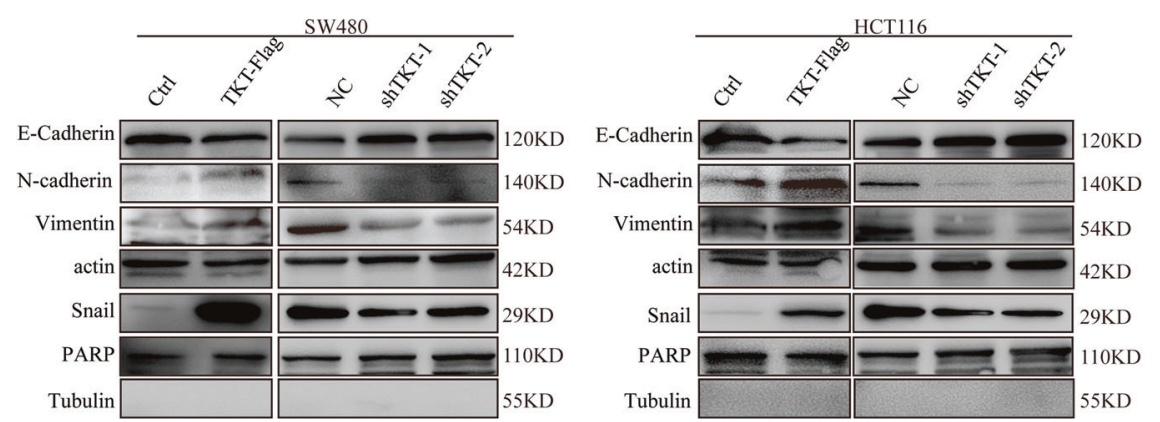

Fig. 4 TKT promotes CRC cell metastasis. (A and B) Representative pictures and analysis of lung metastases. ${ }^{*} P<0.05,{ }^{* *} P<0.01,{ }^{* * *} P<0.001$. (C and D) Corresponding H\&E-stained lung. The arrow pointed to the metastasis. (E and F) Protein levels of EMT markers in SW480 and HCT116. ${ }^{*} P<0.05,{ }^{* *} P<0.01,{ }^{* * *} P<0.001$.

results indicated that the regulation of TKT on glycolysis was dependent on AKT phosphorylation,

\section{Regulation of TKT to AKT phosphorylation and aerobic glycolysis was dependent on GRP78}

On the basis of the above results, we next determined whether GRP78 participated in the regulation of AKT phosphorylation by TKT. We verified the interaction between GRP78 and TKT by using coimmunoprecipitation in SW480 and HCT116 cells (Fig. 7A, B). Then, we found that the overexpression of TKT and knockdown of GRP78 resulted in decreased AKT phosphorylation (Fig. 7C). Moreover, with the knockdown of GRP78, the phenomenon of enhanced glycolysis, glucose consumption, F6P, and lactic acid production, as well as the expression of Glut1 and LDHA caused by the overexpression of TKT was also reversed (Fig. 7D-I).

To validate whether the interaction of TKT and GRP78 could result in functional consequences, we investigated the role of GRP78 in CRC migration and invasion. The results demonstrated that overexpression of TKT and knockdown of GRP78 lead to weakened migration and invasion ability (Fig. 7J-M). These data suggested that TKT regulated the metastasis of CRC cells by binding to GRP78, which affected AKT phosphorylation, which in turn affects cell glycolysis (Fig. S4B).

\section{DISCUSSION}

In the traditional concept, the role of TKT is mainly to participate in PPP and de novo nucleotide biosynthesis. As an important member of the PPP, TKT has been found highly expressed and plays an important role in tumor development. In our previous studies, we found that the incidence of liver cancer was decreased in liver-specific TKT knockout mice compared with control littermates. This observation was explained by TKT absence in hepatocytes protected the liver from DNA damage which is induced by diethylnitrosamine (DEN) [27]. Additionally, the metabolomic analysis suggested that loss of TKT caused a decreased bile acid. Moreover, we confirmed that TKT entered the nucleus by interacting with STAT1, then inhibited FXR expression via enhancing the interaction between HDAC3 and FXR promoter [23]. However, the efficacy of TKT in colorectal cancer has not yet been reported.

In most of the existing studies, TKT mainly plays its role through directly generating ribose 5 -phosphate, providing raw materials for the rapid proliferation of cells. We do not deny that TKT can work in the metastasis of colorectal cancer through the PPP, but our focus is whether TKT can regulate colorectal cancer metastasis in other ways besides PPP. We found for the first time that the expression of TKT in CRC was significantly higher than that in adjacent tissues. Moreover, we demonstrated that high expression of TKT expression was positively correlated with the depth of invasion, LNM, distant metastasis, TNM stage, OS, and DFS. Additionally, the data also confirmed that high TKT expression was an independent indicator for CRC prognostic. These data demonstrate that TKT plays a vital role in colorectal cancer metastasis.

Metastasis is an important cause of death in CRC patients [28]. Previous studies have confirmed that TKT is related to tumor cell growth and metastasis $[18,19,21]$. We found that TKT promoted the proliferation of CRC cells and increased CRC migration and invasion abilities. We also demonstrated that TKT promoted the lung metastasis of CRC cells in vivo. These data clarified that TKT plays an important role in CRC development.

In addition, the present study elucidated how TKT affects the development of colorectal cancer. GRP78 plays an important role in the proliferation, invasion, and metastasis of cancer cells such as prostate, endometrial, and CRC [26, 29-32]. The relationship between GRP78 and AKT has been proven [33], AKT phosphorylation can be regulated by GRP78 [34]. Moreover, AKT activation was 
A
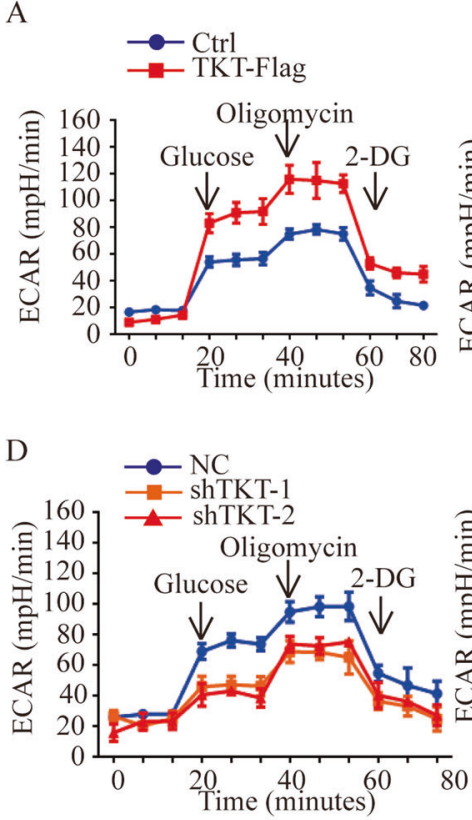

G

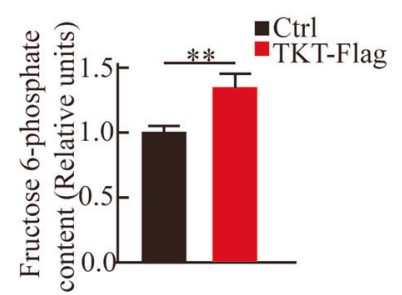

J

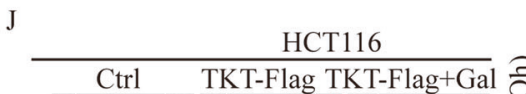

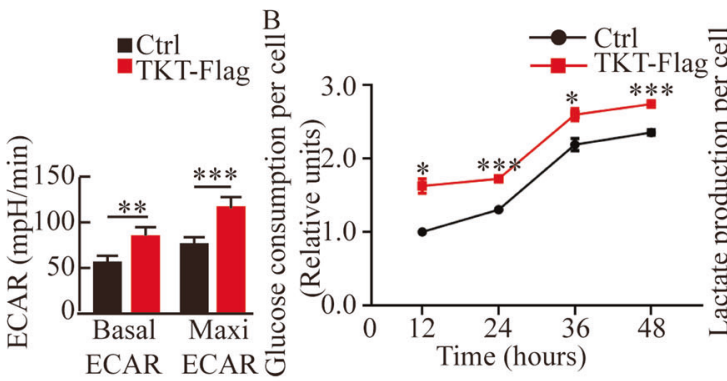
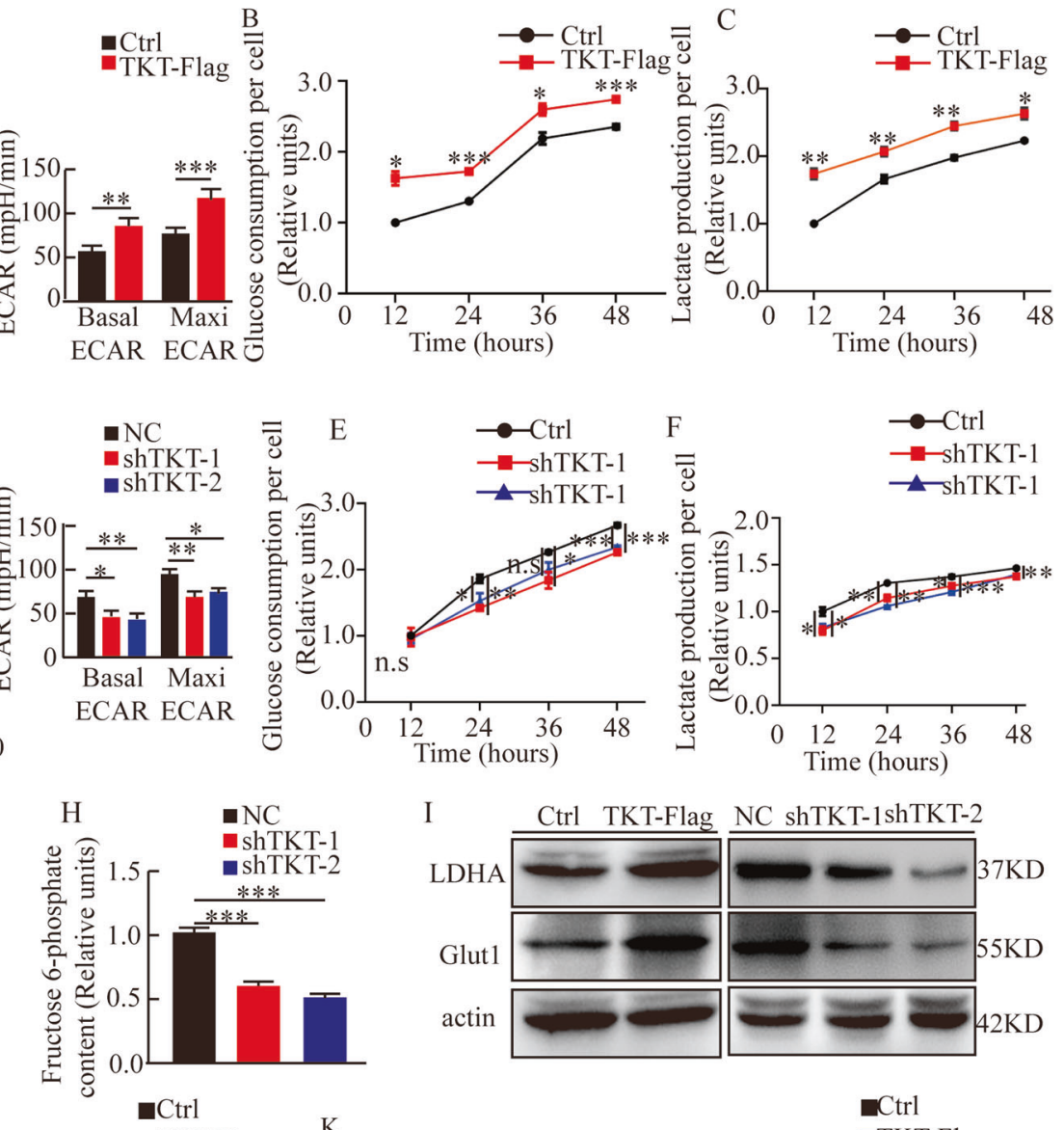

$\square$ Ctrl
$\square$ TKT-Flag K
$\square$ TKT-Flag+Gal

Ctrl

-TKT-Flag

-TKT-Flag+Gal

Ctrl

SW480
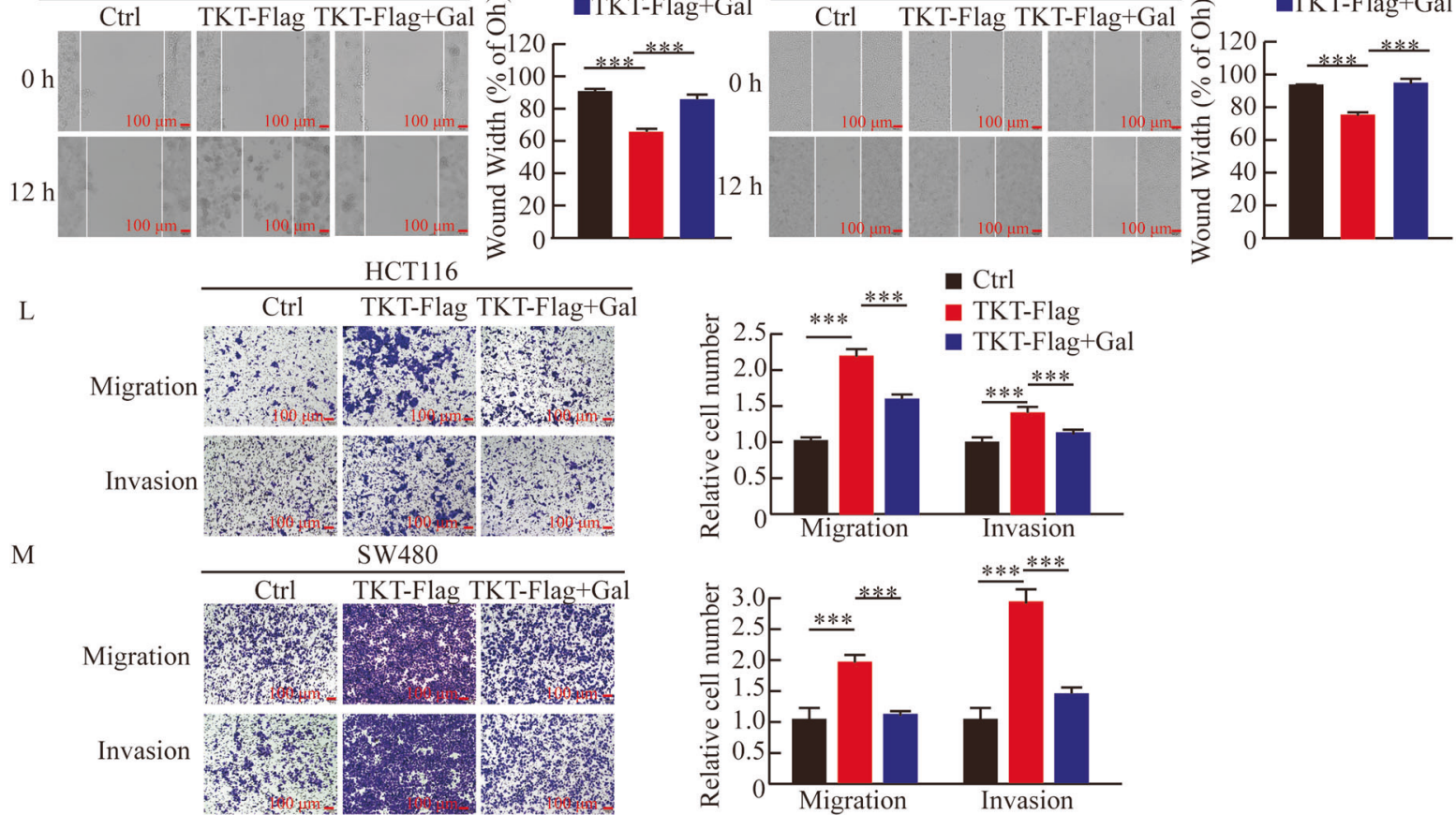

Fig. 5 TKT promoted CRC cells metastasis through activating aerobic glycolysis. (A) Seahorse assays showed that TKT overexpression significantly promoted aerobic glycolysis. Left, representative curve, Right, quantification of three independent experiment of basal ECAR and maxi ECAR. (B-C) TKT promoted glucose consumption and lactate production in HCT116. (D) Seahorse assays showed that TKT knockdown significantly inhibited aerobic glycolysis, Left, representative curve, Right, quantification of three independent experiment of basal ECAR and maxi ECAR. (E-F) Relative glucose consumption and lactate production were detected in HCT116. (G-H) The content of F6P was detected in HCT116. (I) TKT promoted the expression of Glut1 and LDHA. (J-M) Wound-healing, migration and matrigel invasion assays were performed in HCT116 and SW480, ${ }^{*} P<0.05,{ }^{* *} P<0.01,{ }^{* * *} P<0.001$. 
A

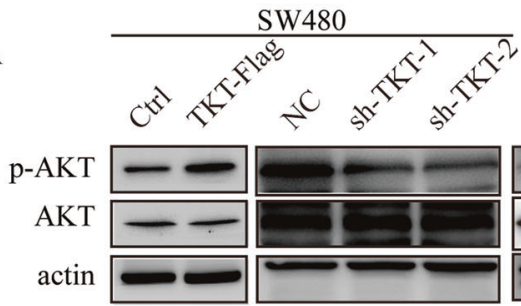

C

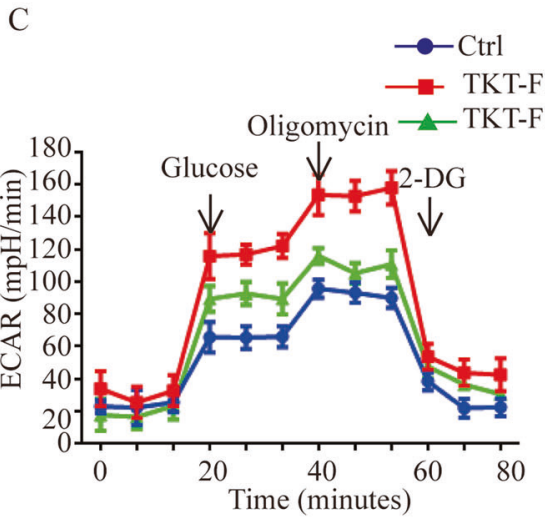

E

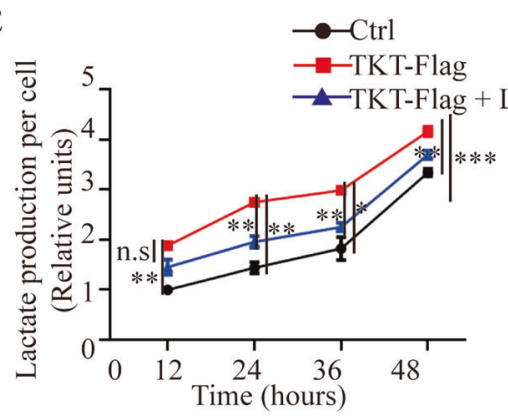

$\mathrm{H}$
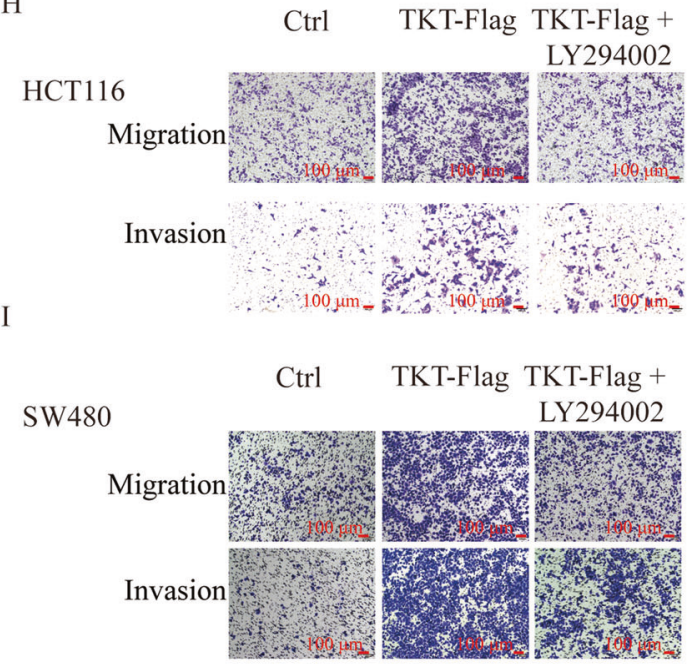

HCT-116
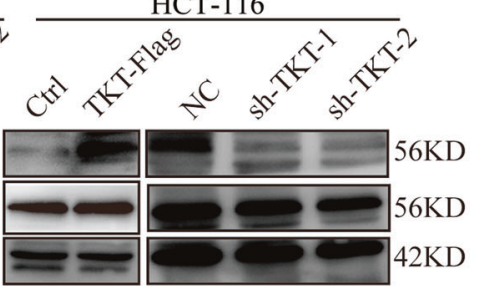

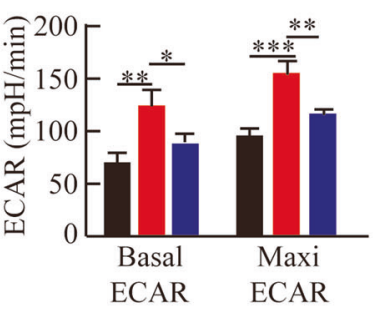

F

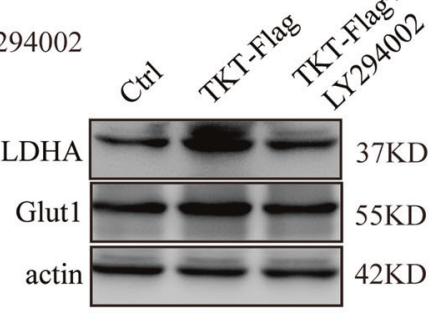

B

D

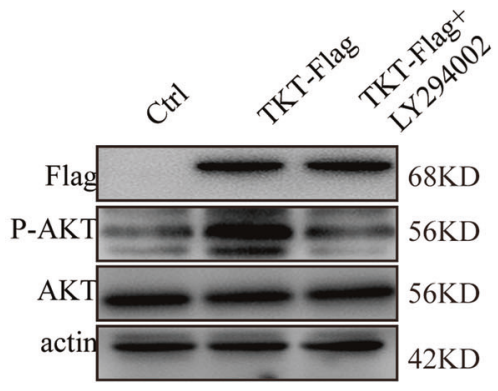

$\rightarrow$ Ctrl

$\rightarrow$-TKT-Flag

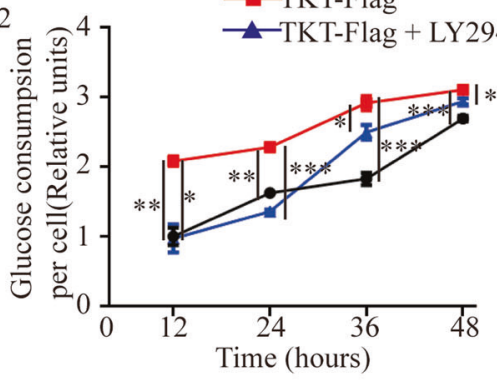

G

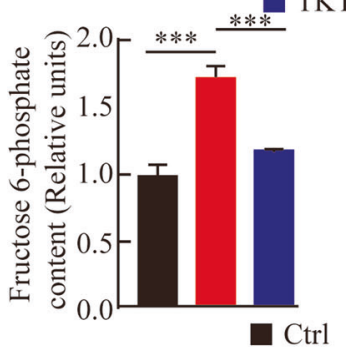

Ctrl

- TKT-Flag

TKT-Flag + LY294002

- TKT-Flag

口 TKT-Flag + LY294002

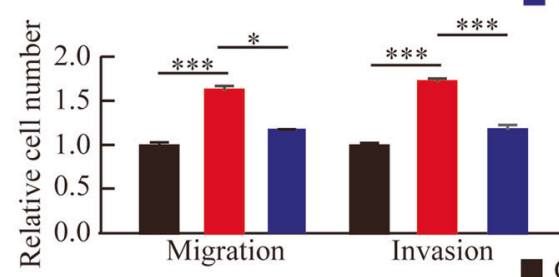

口 Ctrl

TKT-Flag

口 TKT-Flag + LY294002

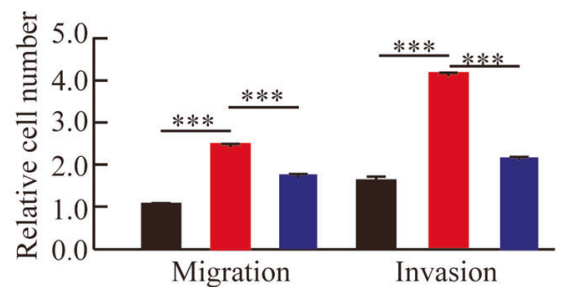

Fig. 6 TKT promotes aerobic glycolysis of CRC cells by promoting AKT phosphorylation. (A) Expressions of AKT phosphorylation in SW480 and HCT116 cells with TKT OE or KD. (B) LY294002 effectively inhibited AKT phosphorylation in HCT116. (C) The regulation of glycolysis by TKT is dependent on AKT phosphorylation, Left, representative curve, Right, quantification of three independent experiment of basal ECAR and maxi ECAR. (D-E) Relative glucose consumption and lactate production were detected in HCT116. (F) Detecting the protein levels of LDHA and Glut1 by using Western blot. (G) The content of F6P was detected in HCT116. (H-I) Migration and invasion detection with AKT phosphorylation inhibitor (LY294002). ${ }^{*} P<0.05,{ }^{* *} P<0.01,{ }^{* * *} P<0.001$. 

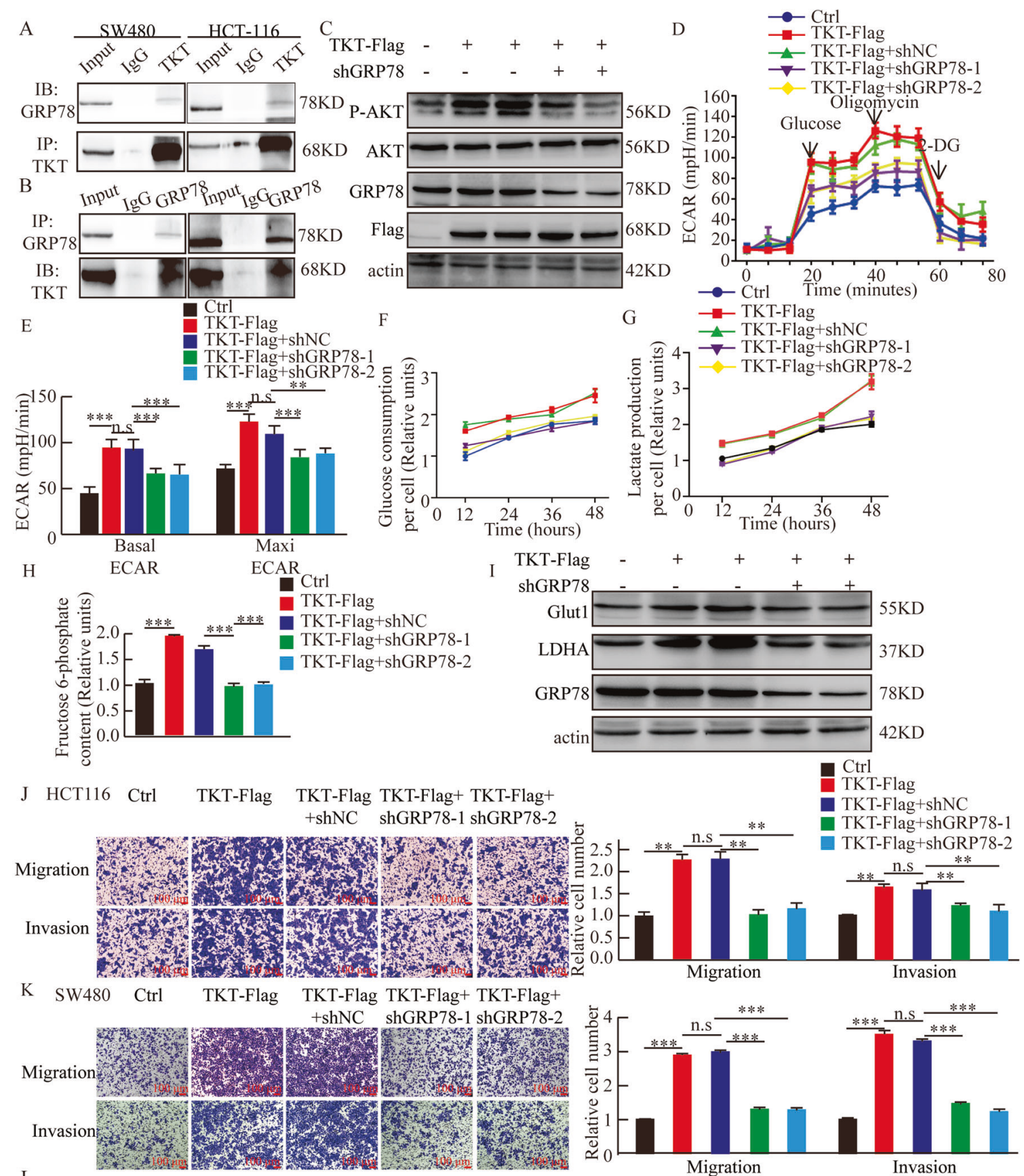

$\mathrm{L}$

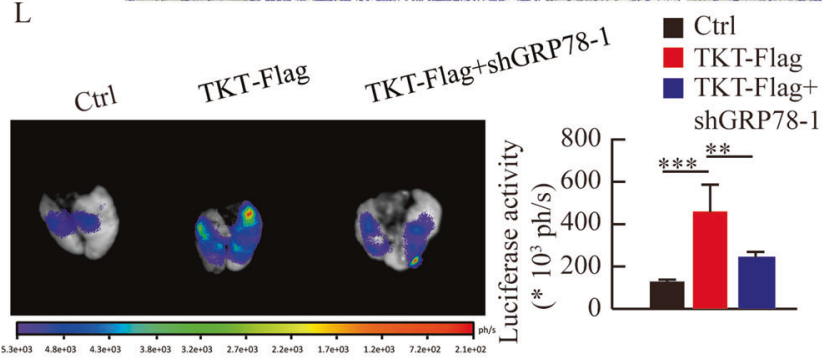

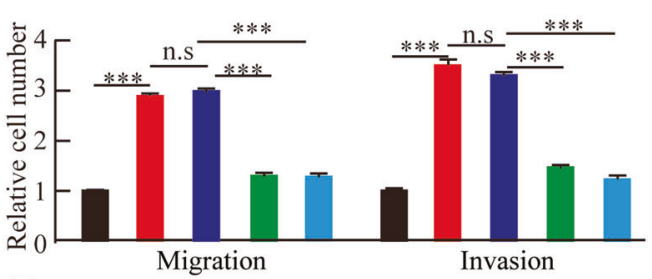

M TKT-Flag+

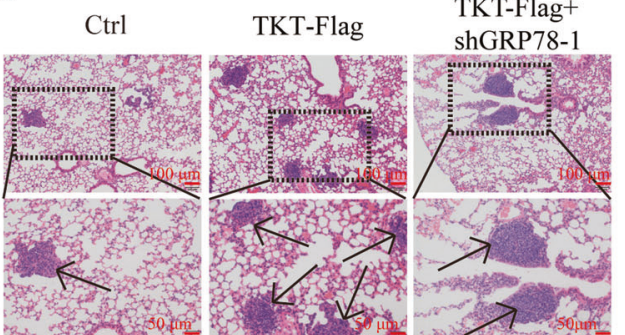

closely related to tumor glycolysis and metastasis [35]. In this work, our data demonstrated that TKT promoted AKT phosphorylation. However, this regulatory effect disappeared with the knockdown of GRP78. We also revealed that blocking GRP78 could significantly reduce TKT-induced CRC cell migration and invasion, which was consistent with the result using the AKT inhibitor LY294002. These data suggested that reducing the expression of TKT could effectively inhibit CRC metastasis. 
Fig. 7 Regulation of TKT to AKT phosphorylation and aerobic glycolysis is dependent on GRP78. (A-B) Endogenous TKT coimmunoprecipitates with GRP78 in SW480 and HCT116. (C) The regulation of TKT to AKT phosphorylation was dependent on GRP78. (D-E) Regulation of TKT to aerobic glycolysis is dependent on GRP78. (F) Relative glucose consumption was determined in HCT116 cells. $12 \mathrm{~h}$ : Ctrl vs. TKT-Flag: *; TKT-Flag vs TKT-Flag + NC: n.s; TKT + NC vs TKT + shGRP78-1: **, TKT + NC vs TKT + shGRP78-2: **. 24 h: Ctrl vs. TKT-Flag: **; TKT-Flag vs TKT-Flag +NC: n.s; TKT + NC vs TKT + shGRP78-1: *, TKT + NC vs TKT + shGRP78-2: *. 36 h: Ctrl vs. TKT-Flag: ${ }^{*} ;$ TKT-Flag vs TKT-Flag +NC: n.s; TKT + NC vs TKT + shGRP78-1: *, TKT + NC vs TKT + shGRP78-2: *. 48 h: Ctrl vs. TKT-Flag: *; TKT-Flag vs TKT-Flag +NC: n.s; TKT + NC vs TKT + shGRP78-1: **, TKT + NC vs TKT + shGRP78-2: *. (G) Relative lactate productionwas determined in HCT116 cells. 12 h: Ctrl vs. TKT-Flag: *; TKT-Flag vs TKT-Flag + NC: n.s; TKT + NC vs TKT + shGRP78-1: **, TKT + NC vs TKT + shGRP78-2: *24 h: Ctrl vs. TKT-Flag: **; TKT-Flag vs TKTFlag +NC: n.s; TKT + NC vs TKT + shGRP78-1: *, TKT + NC vs TKT + shGRP78-2: *. 36 h: Ctrl vs. TKT-Flag: **; TKT-Flag vs TKT-Flag +NC: n.s; TKT + NC vs TKT + shGRP78-1: **, TKT + NC vs TKT + shGRP78-2: **. 48 h: Ctrl vs. TKT-Flag: *; TKT-Flag vs TKT-Flag +NC: n.s; TKT + NC vs TKT + shGRP78-1: **, TKT + NC vs TKT + shGRP78-2: *. (H) The content of F6P was detected in HCT116. (I) Western blot analysis about the expression of Glut1 and LDHA. (J-K) Migration and invasion detection with TKT overexpression and GRP78 knockdown. (L) Representative pictures and analysis of lung metastases in mice. (M) Corresponding H\&E -stained lung sections. Arrows denote lung metastasis. ${ }^{*} P<0.05,{ }^{* *} P<0.01,{ }^{* * *} P<$ 0.001 .

\section{MATERIALS AND METHODS}

\section{Patients and samples collection}

We collected the TMAs (568 pairs of colorectal cancer and adjacent tissues) from the Affiliated Hospital of Xuzhou Medical University. All the patients underwent radical surgery from April 2010 to March 2015. Clinical patient information was provided by the Affiliated Hospital of Xuzhou Medical University.

\section{Cell culture and treatment}

The colorectal cancer cell lines was purchased from the Chinese Academy of Sciences Cell Bank. SW480 and HCT116 were cultured in 1640/DMEM medium. All medium are supplemented with $10 \%$ fetal bovine serum, $1 \%$ penicillin $(100 \mathrm{U} / \mathrm{ml})$, and $1 \%$ streptomycin $(100 \mu \mathrm{g} / \mathrm{ml})$, the culture instrument is a $37^{\circ} \mathrm{C}$ incubator containing $5 \% \mathrm{CO}_{2}$.

\section{Antibodies}

The western blot method was indicated in the reference [36]. The antibodies against TKT, GRP78, Actin were purchased from Proteintech (11039-1-AP, 11587-1-AP, 20536-1-AP). E-cadherin, N-cadherin were purchased from BD Biosciences $(610181,610920)$. Snail (A11794, Abclonal), Glut1 (73015, CST), LDHA (3582, CST).

\section{Immunohistochemistry (IHC)}

The IHC method is shown as previously reported [37]. The dilution ratio of TKT antibody is 1:100.

\section{IHC scoring method}

Two pathologists scored TMA separately and all discrepancies that were resolved after discussion. TKT staining score was determined by combining the rate of positive cells and depending on the IRS. TKT immunostaining intensity score was classified as: negative: $0-3$, weak: 1 , moderate: 2 , strong: 3$)$; the percentage of positive cells was graded as $1(0-25 \%), 2$ $(26-50 \%), 3(51-75 \%)$, and $4(76-100 \%)$. According to IRS, TKT expression levels were classified as follows:low: 0-6, high: 8-12.

\section{Real-time cellular analysis (RTCA)}

The RTCA experiment was carried out according to the manufacturer's instructions (ACEA Biosciences). $0.5 \times 10^{4}$ cells $/ 200 \mathrm{ml}$ medium were seeded in each well, and monitored every $15 \mathrm{~min}$. Experiments about proliferation and migration were done for $96 \mathrm{~h}$ and $36 \mathrm{~h}$, invasion assays were done for $48 \mathrm{~h}$.

\section{Cell migration, invasion, and wound-healing assays}

Cell migration and invasion assays were performed as previously described [38]. For the migration assay, placed the transwell chambers $(8.0 \mu \mathrm{m}$, Corning, NY, USA.) in a 24-well plate with complete medium, then $2 \times 10^{5}$ cells $/ 100 \mu \mathrm{l}$ serum-free median planted to chambers, and incubated for $48 \mathrm{~h}$. For the invasion assay, added matrigel (BD Biosciences) to chambers, then $4 \times 10^{5}$ cells were seeded into chambers after $4 \mathrm{~h}$, and incubated for $48 \mathrm{~h}$.

We seeded cells in 6-well plates and cultivated until the density reaches $90 \%$ confluence. Then, used a sterile $10 \mu \mathrm{l}$ pipette tip to scrathed each well, washed away floating cells, and then added medium with $1 \%$ FBS and photographed at 0 and $24 \mathrm{~h}$.

\section{Stable cell line generation}

We constructed the pCDH-CMV-MCS-EF1-GreenPuro-CD513B-TKT lentivirus plasmid, and purchased TKT and GRP78 short hairpin RNA (shRNA) (GenePharma). HCT116 and SW480 stable cell lines were obtained through lentivirus infection and puromysin screening.

The shRNAs against human TKT and GRP78 were purchased from GenePharma Technology (Shanghai, China). shRNAs were listed in below: ShTKT-1:

GCCGCCAAUACAAAGGGUATTUACCCUUUGUAUUGGCGGCTT

ShTKT-2:

CCGGCAAAUACUUCGACAATTUUGUCGAAGUAUUUGCCGGTT

ShGRP78-1:

GAGGUGUCAUGACCAAACUTTAGUUUGGUCAUGACACCUCTT

ShGRP78-2:

GGGCAAAGAUGUCAGGAAATTUUUCCUGACAUCUUUGCCCTT

\section{Proliferation and colony formation assays}

We used CCK-8 experiments to detect cell proliferation ability according to manufacturer's protocol (Dojindo, Japan). Colony formation assay was performed in six-well plates, one thousand cells were seeded with complete medium, and stained with $0.1 \%$ crystal violet after 14 days, then counted the colony number.

Seahorse assays, glucose uptake, F6P, and lactate production We used Seahorse Bioscience Extracellular Flux Analyzer to detect glycosis via measuring extracellular acidification rate (ECAR) in real time. Basal ECAR is indicated by ECAR in the presence of glucose. Maxi ECAR is indicated by ECAR in the presence of Oligomycin.

Cells were plated in six-well plates. Culture medium was collected at $12 \mathrm{~h}, 24 \mathrm{~h}, 36 \mathrm{~h}$, and $48 \mathrm{~h}$. We used glucose and lactate assay kit (Shanghai Rongsheng Biotech, Shanghai, China) to measure the content of glucose and lactate following manufacturer's instructions.

The content of F6P was measured by using the fructose-6-phosphate assay kit (MAK020, Sigma, USA).

\section{Animal work}

BALB/c nude mice (Male, 8 weeks old) were purchased from Jicui Laboratory Animal Technology (Nanjing, China). All animal experiments were approved by the Experimental Animal Center of Xuzhou Medical University. Injected HCT116-Luc-shNC, HCT116-Luc-shTKT-1, HCT116-LucCtrl, and HCT116-Luc-TKT-Flag cells $\left(5 \times 10^{6}\right)$ into mice through the tail vein, and the fluorescence intensity in the mice was measured by live imaging after 8 weeks.

We injected HCT116-Ctrl, HCT116-TKT-Flag cells $\left(2 \times 10^{6}\right)$ into mice subcutaneously, then measured the length $(\mathrm{L})$ and width $(\mathrm{W})$ of the tumor and calculated with the following formula: Tumor volume $=\left(L \times W^{2}\right) / 2$ after 6 weeks.

\section{Statistical analysis}

Statistical analysis were conducted using GraphPad Prism 7.0. Single-factor analysis for three groups were calculated using one-way ANOVA. Analysis for two groups were analyzed by two-tailed Student's $t$-test. Data were presented as mean $\pm \mathrm{SEM}$, and the bar indicated the mean. $P<0.05$ is statistically significant. 


\section{DATA AVAILABILITY}

The datasets are available from the corresponding author on reasonable request.

\section{REFERENCES}

1. Ganapathy-Kanniappan S, Geschwind JF. Tumor glycolysis as a target for cancer therapy: progress and prospects. Mol Cancer. 2013;12:152.

2. Corbet $C$, Feron $O$. Tumour acidosis: from the passenger to the driver's seat. Nat Rev Cancer. 2017;17:577-93.

3. Lunt SY, Vander, Heiden MG. Aerobic glycolysis: meeting the metabolic requirements of cell proliferation. Annu Rev Cell Dev Biol. 2011;27:441-64.

4. Brizel DM, Schroeder T, Scher RL, Walenta S, Clough RW, Dewhirst MW, et al. Elevated tumor lactate concentrations predict for an increased risk of metastases in head-and-neck cancer. Int J Radiat Oncol Biol Phys. 2001;51:349-53.

5. Walenta S, Voelxen NF, Mueller-Klieser W. Lactate-An Integrative Mirror of Cancer Metabolism. Recent Results Cancer Res. 2016;207:23-37.

6. Walenta S, Wetterling M, Lehrke M, Schwickert G, Sundfor K, Rofstad EK, et al. High lactate levels predict likelihood of metastases, tumor recurrence, and restricted patient survival in human cervical cancers. Cancer Res. 2000;60:916-21.

7. Siegel RL, Miller KD, Goding Sauer A, Fedewa SA, Butterly LF, Anderson JC, et al. Colorectal cancer statistics, 2020. CA Cancer J Clin. 2020;70:145-64.

8. Chen W, Zheng R, Baade PD, Zhang S, Zeng H, Bray $F$, et al. Cancer statistics in China, 2015. CA Cancer J Clin. 2016;66:115-32.

9. Andre N, Schmiegel W. Chemoradiotherapy for colorectal cancer. Gut 2005;54:1194-202.

10. Coy JF, Dubel S, Kioschis P, Thomas K, Micklem G, Delius H, et al. Molecular cloning of tissue-specific transcripts of a transketolase-related gene: implications for the evolution of new vertebrate genes. Genomics 1996;32:309-16.

11. Schenk G, Duggleby RG, Nixon PF. Properties and functions of the thiamin diphosphate dependent enzyme transketolase. Int J Biochem Cell Biol. 1998;30:1297-318.

12. Zhao J, Zhong CJ. A review on research progress of transketolase. Neurosci Bull. 2009;25:94-99.

13. Horecker BL. The pentose phosphate pathway. J Biol Chem. 2002;277:47965-71.

14. da Costa IA, Hennenlotter J, Stuhler V, Kuhs U, Scharpf M, Todenhofer T, et al Transketolase like 1 (TKTL1) expression alterations in prostate cancer tumorigenesis. Urol Oncol. 2018;36:472 e421-472 e427.

15. Zheng X, Li H. TKTL1 modulates the response of paclitaxel-resistant human ovarian cancer cells to paclitaxel. Biochem Biophys Res Commun. 2018;503:572-9.

16. Shi Z, Tang Y, Li K, Fan Q. TKTL1 expression and its downregulation is implicated in cell proliferation inhibition and cell cycle arrest in esophageal squamous cell carcinoma. Tumour Biol. 2015;36:8519-29.

17. Uhlen $M$, Fagerberg L, Hallstrom BM, Lindskog C, Oksvold $P$, Mardinoglu $A$, et al. Proteomics. Tissue-based map of the human proteome. Science. 2015;347:1260419.

18. Xu IM, Lai RK, Lin SH, Tse AP, Chiu DK, Koh HY, et al. Transketolase counteracts oxidative stress to drive cancer development. Proc Natl Acad Sci USA. 2016;113:E725-734.

19. Ricciardelli C, Lokman NA, Cheruvu S, Tan IA, Ween MP, Pyragius CE, et al. Transketolase is upregulated in metastatic peritoneal implants and promotes ovarian cancer cell proliferation. Clin Exp Metastasis. 2015;32:441-55.

20. Wang J, Zhang X, Ma D, Lee WP, Xiao J, Zhao Y, et al. Inhibition of transketolase by oxythiamine altered dynamics of protein signals in pancreatic cancer cells. Exp Hematol Oncol. 2013;2:18.

21. Tseng CW, Kuo WH, Chan SH, Chan HL, Chang KJ, Wang LH. Transketolase Regulates the Metabolic Switch to Control Breast Cancer Cell Metastasis via the alpha-Ketoglutarate Signaling Pathway. Cancer Res. 2018;78:2799-812.

22. Qin Z, Xiang C, Zhong F, Liu Y, Dong Q, Li K, et al. Transketolase (TKT) activity and nuclear localization promote hepatocellular carcinoma in a metabolic and a nonmetabolic manner. J Exp Clin Cancer Res. 2019;38:154.

23. Li M, Zhang $X$, Lu Y, Meng $S$, Quan $H$, Hou $P$, et al. The nuclear translocation of transketolase inhibits the farnesoid receptor expression by promoting the binding of HDAC3 to FXR promoter in hepatocellular carcinoma cell lines. Cell Death Dis. 2020;11:31.

24. Carmeliet $P$, Jain RK. Principles and mechanisms of vessel normalization for cancer and other angiogenic diseases. Nat Rev Drug Disco. 2011;10:417-27.

25. Mao LJ, Yang CH, Fan L, Gao P, Yang DR, Xue BX, et al. SATB1 promotes prostate cancer metastasis by the regulation of epithelial-mesenchymal transition. Biomed Pharmacother. 2016;79:1-8.

26. Zhou X, Xing X, Zhang S, Liu L, Wang C, Li L, et al. Glucose-regulated protein 78 contributes to the proliferation and tumorigenesis of human colorectal carcinoma via AKT and ERK pathways. Oncol Rep. 2016;36:2723-30.

27. Li M, Lu Y, Li Y, Tong L, Gu XC, Meng J, et al. Transketolase Deficiency Protects the Liver from DNA Damage by Increasing Levels of Ribose 5-Phosphate and Nucleotides. Cancer Res. 2019;79:3689-701.

28. Carlomagno C, De Stefano A, Rosanova M, De Falco S, Attademo L, Fiore G, et al. Multiple treatment lines and prognosis in metastatic colorectal cancer patients. Cancer Metastasis Rev. 2019;38:307-13.
29. Kuo LJ, Huang CY, Cheng WL, Hung CS, Wu CT, Lin FY, et al. Glucose-regulated protein 78 mediates the anticancer efficacy of shikonin in hormone-refractory prostate cancer cells. Tumour Biol. 2015;36:5063-70.

30. Lin YG, Shen J, Yoo E, Liu R, Yen HY, Mehta A, et al. Targeting the glucoseregulated protein-78 abrogates Pten-null driven AKT activation and endometrioid tumorigenesis. Oncogene 2015;34:5418-26.

31. Mhaidat NM, Alzoubi KH, Almomani N, Khabour OF. Expression of glucose regulated protein 78 (GRP78) determines colorectal cancer response to chemotherapy. Cancer Biomark. 2015;15:197-203.

32. Wu CT, Wang WC, Chen MF, Su HY, Chen WY, Wu CH, et al. Glucose-regulated protein 78 mediates hormone-independent prostate cancer progression and metastasis through maspin and COX-2 expression. Tumour Biol. 2014;35:195-204.

33. Xie J, Tao ZH, Zhao J, Li T, Wu ZH, Zhang JF, et al. Glucose regulated protein 78 (GRP78) inhibits apoptosis and attentinutes chemosensitivity of gemcitabine in breast cancer cell via AKT/mitochondrial apoptotic pathway. Biochem Biophys Res Commun. 2016;474:612-9.

34. Lin Y, Wang Z, Liu L, Chen L. Akt is the downstream target of GRP78 in mediating cisplatin resistance in ER stress-tolerant human lung cancer cells. Lung Cancer. 2011;71:291-7.

35. Fu QF, Liu Y, Fan Y, Hua SN, Qu HY, Dong SW, et al. Alpha-enolase promotes cell glycolysis, growth, migration, and invasion in non-small cell lung cancer through FAK-mediated PI3K/AKT pathway. J Hematol Oncol. 2015;8:22.

36. Hou P, Li L, Chen F, Chen Y, Liu H, Li J, et al. PTBP3-Mediated Regulation of ZEB1 mRNA Stability Promotes Epithelial-Mesenchymal Transition in Breast Cancer. Cancer Res. 2018;78:387-98.

37. Hou PF, Jiang T, Chen F, Shi PC, Li HQ, Bai J, et al. KIF4A facilitates cell proliferation via induction of p21-mediated cell cycle progression and promotes metastasis in colorectal cancer. Cell Death Dis. 2018;9:477.

38. Hou P, Chen F, Yong H, Lin T, Li J, Pan Y, et al. PTBP3 contributes to colorectal cancer growth and metastasis via translational activation of HIF-1alpha. J Exp Clin Cancer Res. 2019:38:301.

\section{ACKNOWLEDGEMENTS}

We sincerely appreciate researchers who worked for this experiment. This work was supported by grants from the National Natural Science Foundation of China (No. 82002478, 82072649, 81872304), Jiangsu Province Natural Science Foundation (BK20201010, BK20180989, BK20170261), the OutstandingYouth Foundation of Jiangsu Province (BK20200046), the Science and Technology Project of Xuzhou (KC20100), the Postdoctoral Science Foundation of China (2019M651813), the National science research in Universities of Jiangsu Province (20KJB320031).

\section{AUTHOR CONTRIBUTIONS}

$M L L, J B$, and JNZ provided study concept and design. MLL, XZ, JX collected and analyzed the data. PFH and ZWL interpreted the data. MLL, XZ, JX, SXQ, and SFC performed the experiments. $\mathrm{HMY}, \mathrm{PFQ}$, and JX collected the patients' samples. MLL wrote the manuscript. All authors approved the final version of manuscript.

\section{CONFLICT OF INTEREST}

The authors declare no competing interests.

\section{ETHICS APPROVAL AND CONSENT TO PARTICIPATE}

All subjects obtained informed consent. The Ethics approval for human subjects was provided by the ethics committee of the Affiliated Hospital of Xuzhou Medical University.

\section{ADDITIONAL INFORMATION}

Supplementary information The online version contains supplementary material available at https://doi.org/10.1038/s41419-022-04575-5.

Correspondence and requests for materials should be addressed to Junnian Zheng or Jin Bai.

Reprints and permission information is available at http://www.nature.com/ reprints

Publisher's note Springer Nature remains neutral with regard to jurisdictional claims in published maps and institutional affiliations. 
M. Li et al.

(c) (i) Open Access This article is licensed under a Creative Commons Attribution 4.0 International License, which permits use, sharing, adaptation, distribution and reproduction in any medium or format, as long as you give appropriate credit to the original author(s) and the source, provide a link to the Creative Commons license, and indicate if changes were made. The images or other third party material in this article are included in the article's Creative Commons license, unless indicated otherwise in a credit line to the material. If material is not included in the article's Creative Commons license and your intended use is not permitted by statutory regulation or exceeds the permitted use, you will need to obtain permission directly from the copyright holder. To view a copy of this license, visit http://creativecommons. org/licenses/by/4.0/.

(c) The Author(s) 2022 